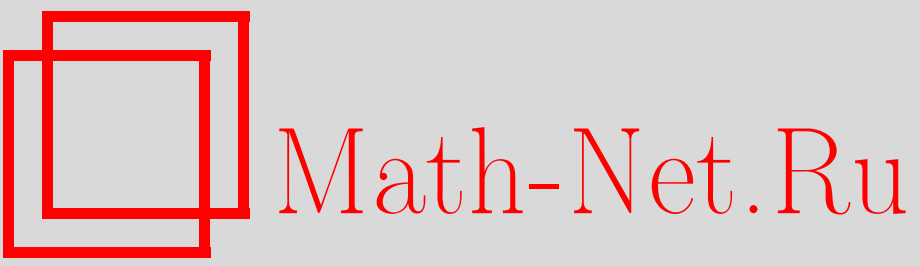

В. А. Бурдов, Некоторые свойства стационарных состояний двухуровневых систем, ТМФ, 1996, том 108, номер 1, 69-78

DOI: https://doi.org/10.4213/tmf1177

Использование Общероссийского математического портала Math-Net.Ru подразумевает, что вы прочитали и согласны с пользовательским соглашением

http://www . mathnet.ru/rus/agreement

Параметры загрузки:

IP: 3.80 .181 .102

26 апреля 2023 г., 18:02:29 


\section{В. А. Бурдов}

\section{НЕКОТОРЫЕ СВОЙСТВА СТАЦИОНАРНЫХ СОСТОЯНИЙ ДВУХУРОВНЕВЫХ СИСТЕМ}

Показано, что существует некая функция $J(x)$, сохраняющая свое значение в любой точке $x$ при произвольных изменениях потенциальной энергии двухуровневой системы. Приведены оценки границ области применимости метода ЛКАО для вычисления волновых функций одномерной двухуровневой системы.

\section{1. ВВЕДЕНИЕ}

Одним из упрошений, возникающих при рассмотрении различных квантовых систем, является двухуровневое приближение, которое позволяет получить наглядные результаты, не прибегая к громоздким вычислениям. Так, например, в работах [1 - 3] с помощью приближения двухуровневой системы исследована многоэлектронная динамика в гетероструктурах на основе квантовых ям.

Расчет волновых функций двухуровневой системы и величины расшепления уровней может быть проведен по методу, который хорошо известен в молекулярной физике (см., например, $[4,5])$ как метод линейной комбинации атомных орбиталей (ЛКАО). В случае двухъямного потенциала волновая функция ищется в виде суперпозиции волновых функций в изолированных ямах, а коэффициенты разложения определяются из условия минимальности энергии данного состояния [6, 7]. При этом, если исходньй потенциал симметричный, волновые функции двух близких уровней имеют вид

$$
\chi_{0}(x)=(\psi(x)+\psi(-x)) / \sqrt{2}, \quad \chi_{1}(x)=(\psi(x)-\psi(-x)) / \sqrt{2} .
$$

Разность энергий двух уровней $\Delta=E_{1}-E_{0}$ определяется при этом интегралом перекрытия волновых функций в изолированных ямах и поэтому является малой. Волновые же функции обладают тем свойством, что их квадраты отличаются на величину, также определяемую произведением $\psi(x) \psi(-x)$. Таким образом, можно считать, что

$$
\left|\chi_{0}^{2}(x)-\chi_{1}^{2}(x)\right|<C_{0} \cdot \Delta
$$

где $C_{0}$ - некоторая размерная постоянная. В квазиклассическом приближении, как показано в [8], величина $\Delta$ экспоненциально мала по параметру $\int_{c}^{d} d x|p(x)| / \hbar$ (где $c$ и $d-$ гранишы классически запрешенной области).

Недостаток такого вариационного метода состоит в том, что с его помощью невозможно оценить величину ошибки, совершаемой при замене истинной волновой функции 
линейной комбинацией волновых функций в изолированных ямах. Задача данной работы состоит как раз в проведении этих оценок, а также в выяснении некоторых закономерностей, присуших волновым функциям двухуровневых систем.

\section{2. ОСНОВНЫЕ УРАВНЕНИЯ ЗАДАЧИ}

Будем рассматривать одномерную квантовую систему с дискретным спектром собственных значений $E_{n}$ и допустим, что в спектре существуют два близких уровня таких, что

$$
\Delta /\left(E_{2}-E_{1}\right) \rightarrow 0
$$

Для простоты предположим, что условие (2) выполняется для основного и первого возбужденного состояний. Гамильтониан $\widehat{H}$ и собственные функции стационарных состояний $\chi_{n}(x)$ будем считать известными. При этом мы не будем ограничиваться случаем, когда гамильтониан инвариантен относительно замены $x \rightarrow-x$, а исследуем более общую ситуацию.

Рассмотрим также другую систему, близкую к исходной, с гамильтонианом

$$
\widehat{H}_{\mu}=\widehat{H}+\mu \cdot U(x),
$$

где $U(x)$ - произвольная функция, описывающая возмущение, а $\mu$-некоторый параметр. Относительно функции $U(x)$ будем предполагать лиш то, что она не меняет граничных условий, накладываемых на волновые функции системы. В этом случае спектр системы (3) и ее волновые функции могут быть описаны рядами теории возмущений (см., например, [8])

$$
\begin{aligned}
& E_{n}(\mu)=E_{n}+\mu U_{n n}+O\left(\mu^{2}\right), \\
& \psi_{0}(x)=\chi_{0}(x)-\mu \frac{U_{01}}{\Delta} \chi_{1}(x)-\mu \sum_{k=2}^{\infty} \frac{U_{0 k}}{E_{k}-E_{0}} \chi_{k}(x)+O\left(\mu^{2}\right), \\
& \psi_{1}(x)=\chi_{1}(x)+\mu \frac{U_{01}}{\Delta} \chi_{0}(x)-\mu \sum_{k=2}^{\infty} \frac{U_{1 k}}{E_{k}-E_{1}} \chi_{k}(x)+O\left(\mu^{2}\right),
\end{aligned}
$$

где введено стандартное обозначение $U_{i j}$ для матричного элемента возмушения, а символом $O\left(\mu^{2}\right)$ обозначены все члены ряда порядка $\mu^{2}$ и выше.

Домножим уравнение Шредингера для волновой функции $\psi_{0}(x)$ на $\psi_{1}(x)$, а уравнение для $\psi_{1}(x)$ на $\psi_{0}(x)$, вычтем из одного другое и проинтегрируем в пределах от $x$ до $\infty$. Будем иметь

$$
\psi_{0}(x) \psi_{1}^{\prime}(x)-\psi_{1}(x) \psi_{0}^{\prime}(x)=2 m \Delta(\mu) \hbar^{2} \int_{x}^{\infty} d y \psi_{0}(y) \psi_{1}(y)
$$

где $\Delta(\mu)=E_{1}(\mu)-E_{0}(\mu)$, а штрих означает дифференцирование по координате. Полагая в уравнении (5) параметр $\mu$ равным нулю, получим в точности такое же уравнение, 
связьвающее волновые функции исходной системы $\chi_{0}(x)$ и $\chi_{1}(x)$ с малой разницей энергий $\Delta$. Дифференцируя (5) по $\mu$ в точке $\mu=0$, найдем

$$
\begin{gathered}
\left(U_{11}-U_{00}\right) \int_{x}^{\infty} d y \chi_{0}(y) \chi_{1}(y)-U_{01} \int_{x}^{\infty} d y\left(\chi_{1}^{2}(y)-\chi_{0}^{2}(y)\right)= \\
=\frac{\hbar^{2}}{2 m} \sum_{k=2}^{\infty}\left\{\frac{U_{0 k}}{E_{k}-E_{1}}\left(\chi_{1}(x) \chi_{k}^{\prime}(x)-\chi_{k}(x) \chi_{1}^{\prime}(x)\right)-\right. \\
\left.-\frac{U_{1 k}}{E_{k}-E_{0}}\left(\chi_{0}(x) \chi_{k}^{\prime}(x)-\chi_{k}(x) \chi_{0}^{\prime}(x)\right)\right\} .
\end{gathered}
$$

В дальнейшем в целях сокрашения записи левую часть равенства (6) будем обозначать как $J(x)$ и примем к сведению, что функция $J(x)$, определяемая волновыми функциями двух близких уровней $\chi_{0,1}(x)$, является еше и функционалом относительно произвольной функции $U(x)$. Полученные уравнения (5) и (6) помогут нам впоследствии исследовать некоторые характерные особенности волновых функций двухуровневых систем и произвести соответствующие оценки.

\section{3. ИНВАРИАНТ ДВУХУРОВНЕВОЙ СИСТЕМЫ}

Остановимся теперь на одном важном свойстве функции $J(x)$, предварительно заметив следуюшее. Поскольку для рассматриваемой нами квантовой системы с потенциальной энергией $V(x)$ выполняется условие $(2)$, любое изменение потенциальной энергии (сопоставимое с разницей энергий $\Delta$ ) не приводит к каким-либо качественным изменениям в энергетическом спектре системы. В то же время новые волновые функции основного и первого возбужденного состояний могут при этом сушественно отличаться от старых.

Вычислим новые волновые функции $\widetilde{\chi}_{0,1}(x)$, отвечающие потенциальной энергии

$$
\widetilde{V}(x)=V(x)+W(x) .
$$

На величину возмущения $W(x)$ наложим условие малости

$$
W_{i j} /\left(E_{2}-E_{1}\right) \rightarrow 0
$$

однако предположим, что по сравнению с величиной $\Delta$ возмушение может изменяться в широких пределах.

При выполнении условия (7) для вычисления волновых функций и энергетического спектра $\widetilde{E}_{0,1}$ двухуровневой системы можно применить основное приближение теории возмушений для вырожденных состояний, что дает для разности энергий двух уровней

$$
\delta=\widetilde{E}_{1}-\widetilde{E}_{0}=\sqrt{\left(\Delta+W_{11}-W_{00}\right)^{2}+4 W_{01}^{2}} .
$$

В этом выражении, как и в выражении (7), матричные элементы $W_{i j}$ вычислены в старом базисе $\chi_{0,1}(x)$. Для произведения волновых функций и разности их квадратов будем иметь

$$
\begin{gathered}
\tilde{\chi}_{0}(x) \tilde{\chi}_{1}(x)=\frac{\Delta+W_{11}-W_{00}}{\delta} \chi_{0}(x) \chi_{1}(x)-\frac{W_{01}}{\delta}\left(\chi_{1}^{2}(x)-\chi_{0}^{2}(x)\right), \\
\tilde{\chi}_{1}^{2}(x)-\tilde{\chi}_{0}^{2}(x)=\frac{\Delta+W_{11}-W_{00}}{\delta}\left(\chi_{1}^{2}(x)-\chi_{0}^{2}(x)\right)+4 \frac{W_{01}}{\delta} \chi_{0}(x) \chi_{1}(x) .
\end{gathered}
$$


Определим функцию $\widetilde{J}(x)$ в произвольном базисе так же, как это было сделано в базисе $\chi_{0,1}(x)$,

$$
\widetilde{J}(x)=\left(\widetilde{U}_{11}-\widetilde{U}_{00}\right) \int_{x}^{\infty} d y \widetilde{\chi}_{0}(y) \widetilde{\chi}_{1}(y)-\widetilde{U}_{01} \int_{x}^{\infty} d y\left(\widetilde{\chi}_{1}^{2}(y)-\widetilde{\chi}_{0}^{2}(y)\right),
$$

где $\widetilde{U}_{i j}$ - матричные элементы произвольной функции $U(x)$ в новом базисе $\widetilde{\chi}_{0,1}$. Подставляя в определение (9) соотношения (8), получим

$$
\widetilde{J}(x)=J(x)
$$

Равенство (10) представляет собой своеобразньй “закон сохранения” - функция $J(x)$ оказывается инвариантом двухуровневой системы по отношению к изменениям потенциальной энергии $V(x)$.

Попробуем теперь произвести оценку абсолютной величины функции $J(x)$, которая, как следует из ее определения, задается волновыми функциями, а следовательно, исходным потенциалом системы $V(x)$. Допустим, что потенциал $V(x)$ подобран таким образом, что распределения по координатам в основном и первом возбужденном состояниях отличаются очень незначительно:

$$
\left|\chi_{0}^{2}(x)-\chi_{1}^{2}(x)\right|<C \cdot \epsilon
$$

где $C$ - размерная постоянная, а $\epsilon \rightarrow 0$.

В этом случае, как видно из (8), при $W_{01} \ll \Delta$ новые волновые функции практически не отличаются от старых, и для них также будет выполнено условие (11). Если же реализуется обратное неравенство $W_{01} \gg \Delta$, тогда малым становится уже произведение функций $\widetilde{\chi}_{0}(x) \widetilde{\chi}_{1}(x)$, в то время как разность квадратов никакой малости не содержит. Изменяя потенциал возмушения $W(x)$, можно реализовать любую из этих двух ситуаций или же какую-либо промежуточную, когда ни произведение функций, ни разность их квадратов не являются малыми величинами (так будет, например, если исходный потенциал двухуровневой системы симметричный, а потенциал возмушения антисимметричный и при этом $W_{01} \sim \Delta$ ). Очевидно, справедливо и обратное: всегда можно подобрать такое возмущение для произвольной двухуровневой системы, что новые волновые функции будут подчиняться неравенству (11). Единственное условие, которое должно быть при этом выполнено, - чтобы при изменении потенциальной энергии от $V(x)$ до $V(x)+W(x)$ система всегда оставалась двухуровневой, т.е. $\delta /\left(\widetilde{E}_{2}-\widetilde{E}_{1}\right) \rightarrow 0$, что происходит автоматически при выполнении условия (7). Если же условие (7) нарушается, систему уже нельзя считать двухуровневой и полученные здесь выражения утрачивают смысл. Верхний из двух уровней при этом отшепляется от основного и по мере увеличения возмушения приближается к уровню $\widetilde{E}_{2}$. В какой-то момент, при определенных значениях $W(x)$, уже первый и второй уровни $\left(\widetilde{E}_{1}\right.$ и $\left.\widetilde{E}_{2}\right)$ образуют двухуровневую систему, для описания которой снова можно применить полученные ранее выражения.

Попытаемся теперь определить величину $\epsilon$, введенную в (11), для чего предварительно заметим следуюшее. Поскольку волновая функция основного состояния не должна иметь нулей, а функция $\chi_{1}(x)$ один раз обрашается в нуль, можно сделать вьвод, принимая во внимание неравенство (11), что функция $\chi_{0}(x)$ имеет минимум в некоторой точке 
$x=x_{0}$. Обозначая через $x_{1}$ значение координаты $x$, при котором $\chi_{1}(x)$ обрашается в нуль, найдем из (5), полагая $\mu=0$,

$$
\chi_{0}\left(x_{0}\right) \chi_{1}^{\prime}\left(x_{0}\right)-\chi_{0}\left(x_{1}\right) \chi_{1}^{\prime}\left(x_{1}\right)=\frac{2 m \Delta}{\hbar^{2}} \int_{x_{1}}^{x_{0}} d x \chi_{0}(x) \chi_{1}(x) .
$$

Оценим величину интеграла в правой части (12). Для определенности будем считать, что $x_{0}>x_{1}, \chi_{0}(x)>0$ и $\chi_{1}(x)>0$ при $x>x_{1}$. Следовательно, значение интеграла должно быть меньше произведения $\left(x_{0}-x_{1}\right) \chi_{0}\left(x_{1}\right) \chi_{1}\left(x_{0}\right)$. Учитывая $(11)$, можно записать при $x>x_{1}$

$$
\left|\chi_{0}(x)-\chi_{1}(x)\right|<A \cdot \epsilon,
$$

что дает $\chi_{0}\left(x_{1}\right)<A \cdot \epsilon, \chi_{1}\left(x_{0}\right)<2 A \cdot \epsilon$. Связь между постоянными $A$ и $C$ легко может быть получена с помошью (11) (например, можно выбрать $C=2 A \chi_{\max }$ ). Таким образом, правая часть (12) не превышает значения

$$
\frac{4 m A^{2}}{\hbar^{2}} \Delta \epsilon^{2}\left(x_{0}-x_{1}\right)
$$

и в силу малости полученного выражения справедливы следуюшие приближенные равенства:

$$
\chi_{0}\left(x_{0}\right) \chi_{1}^{\prime}\left(x_{0}\right) \approx \chi_{0}\left(x_{1}\right) \chi_{1}^{\prime}\left(x_{1}\right) \approx \chi_{0}\left(x_{0}\right) \chi_{1}^{\prime}\left(x_{1}\right) \approx m \Delta / \hbar^{2} .
$$

Разложим левую часть (12) в ряд по малой разнице $x_{0}-x_{1}$. Принимая во внимание (13) и вводя обозначение $F=\left|V^{\prime}(z)\right|$, получим следующую оценку:

$$
\left|x_{0}-x_{1}\right|<2 \hbar A \epsilon / \sqrt{m F}
$$

где $z=\left(x_{0}+x_{1}\right) / 2$. Выражение $(14)$ позволит нам в дальнейшем пренебречь разницей $x_{0}-x_{1}$ и считать в главном приближении эти точки совпадающими.

Проведем оценки величины инварианта двухуровневой системы $J(x)$ в точке $x=z$. Учитывая $(6)$ и (14), для $J(z)$ получим

$$
J(z)=-\frac{\hbar^{2}}{2 m} \sum_{k=2}^{\infty}\left\{\frac{U_{0 k}}{E_{k}-E_{1}} \chi_{1}^{\prime}(z) \chi_{k}(z)+\frac{U_{1 k}}{E_{k}-E_{0}} \chi_{0}(z) \chi_{k}^{\prime}(z)\right\}
$$

Значения функций $\chi_{k}(z)$ и их производных, вообще говоря, произвольны и никакой малости не содержат. В свою очередь, $\chi_{0}(z)$ и $\chi_{1}^{\prime}(z)$ связаны соотношением (13) - их произведение пропорционально малой величине $\Delta$.

Для определения $\chi_{0}(z)$ и $\chi_{1}^{\prime}(z)$ поступим следующим образом. Воспользуемся тем обстоятельством, что потенциальная энергия $V(x)$ предполагается непрерьвной функцией вместе со своими производными любого порядка и, следовательно, волновые функции системы будут также дифференцируемы до любого порядка в произвольной точке оси $x$. Будем искать решение уравнения Шредингера для функций $\chi_{0}(x)$ и $\chi_{1}(x)$ в виде ряда Тейлора в окрестности точек $x_{0}$ и $x_{1}$, соответственно. При этом, как нетрудно убедиться непосредственно с помошњю уравнения Шредингера, оказывается, что производные любого порядка (кроме производной первого порядка, которая равна нулю) от функции $\chi_{0}(x)$ в точке $x=x_{0}$ определяются значением самой функции в этой точке. 
Аналогично все производные от функции $\chi_{1}(x)$ в точке $x_{1}$ (кроме производной второго порядка, равной нулю) пропорциональны $\chi_{1}^{\prime}\left(x_{1}\right)$.

Таким образом, волновые функции $\chi_{0}(x)$ и $\chi_{1}(x)$ могут быть представлены в следующем виде:

$$
\chi_{0}(x)=\chi_{0}\left(x_{0}\right) f\left(x-x_{0}\right), \quad \chi_{1}(x)=\chi_{1}^{\prime}\left(x_{1}\right) g\left(x-x_{1}\right),
$$

где $f\left(x-x_{0}\right)$ и $g\left(x-x_{1}\right)$ - некоторые функции, коэффициенты разложения которых в степенной ряд уже не содержат самих волновых функций или их производных в точках $x_{0}$ и $x_{1}$, соответственно.

Теперь воспользуемся ограничительными неравенствами (11) и (14) и, пренебрегая ошибкой порядка $\epsilon$, запишем равенство

$$
\chi_{0}^{2}(z) f^{2}(x-z)=\chi_{1}^{\prime 2}(z) g^{2}(x-z)
$$

которое справедливо в любой точке на оси $x$. Очевидно, функции $f^{2}(x-z)$ и $g^{2}(x-z)$ должны быть одинаковы с точностью до постоянного множителя (обозначим его $q^{2}$ ), что, в свою очередь, приводит к соотношению

$$
\chi_{1}^{\prime}(z)=q \chi_{0}(z)
$$

По своему смыслу произведение $\hbar q$ представляет собой некоторое характерное значение модуля классического импульса частищы в окрестности точки $x=z$ и не зависит от $\epsilon$ (см. приложение).

Значения $\chi_{0}(z)$ и $\chi_{1}^{\prime}(z)$ с учетом $(13)$ и $(17)$ оказываются пропорциональными $\sqrt{\Delta}$ :

$$
\chi_{0}(z)=\sqrt{\frac{m \Delta}{\hbar^{2} q}}, \quad \chi_{1}^{\prime}(z)=\sqrt{\frac{q m \Delta}{\hbar^{2}}},
$$

откуда заключаем, что и вся правая часть (15) имеет тот же порядок малости.

С другой стороны, само определение инварианта $J(x)$ в точке $x=z$ может быть несколько упрощено. Обратим внимание на то, что точка $x=z$ близка с точностью до слагаемых порядка $\epsilon$ к значениям $z_{0}$ и $z_{1}$, которые определяются из соотношений

$$
\int_{z_{0}}^{\infty} d x \chi_{0}^{2}(x)=1 / 2, \quad \int_{z_{1}}^{\infty} d x \chi_{1}^{2}(x)=1 / 2 .
$$

Величина интеграла от разности квадратов волновых функций, входящего в определение $J(z)$, равна

$$
\int_{z}^{\infty} d x\left(\chi_{1}^{2}(x)-\chi_{0}^{2}(x)\right)=\int_{z}^{z_{1}} d x \chi_{1}^{2}(x)+\int_{z_{0}}^{z} d x \chi_{0}^{2}(x)
$$

и, поскольку $\left|z-z_{0,1}\right| \sim \epsilon$, а $\chi_{0}^{2}(z) \sim \chi_{1}^{\prime 2}(z) \sim \Delta$, вторым слагаемым в функции $J(z)$ можно пренебречь по сравнению с первым. Принимая во внимание приближенное равенство

$$
\int_{z}^{\infty} d x \chi_{0}(x) \chi_{1}(x) \approx 1 / 2
$$


будем иметь

$$
J(z)=\frac{U_{11}-U_{00}}{2},
$$

и, следовательно, с помошью (15)и (18) получаем

$$
\int_{-\infty}^{+\infty} d x U(x)\left(\chi_{0}^{2}(x)-\chi_{1}^{2}(x)\right)=\frac{E}{\sqrt{q}} \sqrt{\frac{\Delta}{E}} \sum_{k=2}^{\infty}\left\{\frac{U_{0 k}}{E_{k}-E_{1}} \chi_{k}(z)+\frac{U_{1 k}}{E_{k}-E_{0}} \frac{\chi_{k}^{\prime}(z)}{q}\right\} .
$$

Так как функция $U(x)$ с самого начала предполагалась произвольной, выражение (19) приводит нас к оценке (11), где в качестве малого безразмерного параметра $\epsilon$ можно теперь принять выражение

$$
\epsilon=(\Delta / E)^{1 / 2},
$$

представляюшее собой корень из отношения разности энергий двух близких уровней $\Delta$ к некоторой характерной энергии системы $E=\hbar^{2} q^{2} / m$. Постоянная $C$ в неравенстве (11) зависит от конкретного вида функции $U(x)$. Если выбрать

$$
U(x)=\delta(x-t),
$$

можно получить из (19) выражение непосредственно для разности квадратов волновых функций. Обозначая в этом случае максимальное значение модуля правой части (19) через $C \cdot \epsilon$, определяем постоянную $C$ в (11).

Продолжим теперь вычисление функции $J(x)$. Учитывая определение $J(x)$, с помошью (11) и (20) можно заключить, что в любой точке оси $x$ (а не только в точке $x=z$ ) будет иметь место оценка

$$
|J(x)|<C(x) \cdot \epsilon,
$$

где $C(x)$ - некоторая зависяшая от $x$ размерная функция. Явный вид этой функции нетрудно установить, пользуясь соотношением (19) и определением $J(x)$, однако сейчас в этом нет большой необходимости - для нас важно лишь то, что функция $C(x)$ не зависит от $\epsilon$. Поскольку функция $J(x)$ является инвариантом двухуровневой системы, окончательно можно утверждать, что для любой двухуровневой системы (даже для такой, которая не подчиняется условию (11)) будем иметь оценку

$$
|\widetilde{J}(x)|<C(x) \cdot \epsilon .
$$

Подчеркнем, что в правую часть (21) входят величины, относяшиеся к так называемой исходной двухуровневой системе с потенциалом $V(x)$ и разницей энергий $\Delta$, которая может быть сушественно меньше разницы энергий $\delta$, отвечающей возмушенной двухуровневой системе (см. ниже).

\section{4. СРАВНЕНИЕ С МЕТОДОМ ЛКАО}

Сопоставим оценки, полученные в предыдушем разделе, с оценками метода ЛКАО для разности квадратов волновых функций двух близких уровней в так называемом резонансном случае. С точки зрения метода ЛКАО резонансный случай реализуется, когда энергетические уровни в изолированных ямах совпадают и величина $\delta$ оказывается 
минимальной и равной $\Delta$. Изменение потенциальной энергии на величину $W(x)$ приводит к относительному сдвигу энергетических уровней в изолированных ямах (но при этом все равно $\left.\delta /\left(\widetilde{E}_{2}-\widetilde{E}_{1}\right) \rightarrow 0\right)$, и, если сдвиг достаточно сильный, величина $\delta$ приближается к значению разности энергий уровней в изолированных ямах и становится много больше своего резонансного значения $\Delta$.

Как уже было отмечено во введении, в резонансном случае в приближении ЛКАО всегда реализуется условие (1), т.е. разность квадратов волновых функций ограничена величиной порядка $\epsilon^{2}$, как следует из (20). Если систему вывести из резонанса, сильно раздвинув первоначально близкие уровни в соседних ямах, в условии (1) можно заменить разность квадратов волновых функций их произведением, которое также будет иметь значение, пропорциональное $\epsilon^{2}$, в то время как разность квадратов уже не будет малой.

В нашем случае разность квадратов волновых функций в резонансном приближении, как следует из (19), определяется величиной $\epsilon$. Выход системы из резонанса здесь также ведет к увеличению этой разницы и к уменьшению произведения $\widetilde{\chi}_{0}(x) \tilde{\chi}_{1}(x)$. Однако в отличие от метода ЛКАО, принимая во внимание наличие инварианта (10) и его оценку (21), для произведения волновых функций получаем, как и для разности квадратов, значение порядка $\epsilon$.

Очевидно, неравенства типа (1), которые накладываются на волновые функции метода ЛКАО, являются более сильными, чем неравенство (21), из которого следует в некотором частном случае ограничение (11). Можно использовать эти неравенства для оценки величины ошибки, допускаемой при замене истинных волновых функций системы $\chi_{0,1}(x)$ на волновые функции метода ЛКАО $\chi_{0,1}^{(0)}(x)$. Считая малым отклонение $\chi_{0,1}^{(0)}(x)$ от истинного решения:

$$
\left|\delta_{0,1}(x)\right| \equiv\left|\chi_{0,1}(x)-\chi_{0,1}^{(0)}(x)\right| \ll\left|\chi_{0,1}(x)\right|,
$$

можно получить из (1) и (11), пренебрегая слагаемыми порядка $\epsilon^{2}$, следуюшее неравенство:

$$
\left|\delta_{0}(x)-\delta_{1}(x)\right|<A \cdot \epsilon .
$$

Учитывая, что речь идет о произвольной двухуровневой системе и функции $\delta_{0}(x)$ и $\delta_{1}(x)$ независимы, найдем, что искомая ошибка метода ЛКАО не превышает значения

$$
\left|\delta_{0,1}(x)\right|<A \cdot \epsilon .
$$

Нужно, однако, иметь в виду, что оценки (11) и (22) следует рассматривать как некие предельно возможные значения для произвольной двухуровневой системы, не учитываюшие никаких ее конкретных свойств. Если же обратиться к двухуровневой системе какого-то вполне определенного вида, может оказаться, что неравенства (11) и (22) усилятся (как это имеет место, например, в случае симметричного потенциала с прямоугольным разделительным барьером; здесь разница квадратов волновых функций порядка $\epsilon^{2}$ ), что расширит возможности применения метода ЛКАО для данной системы.

В заключение заметим, что в настоящей работе нигде (кроме приложения) не были использованы конкретный вид потенциальной энергии и структура спектра и волновых функций в изолированных ямах. Единственное положение, на котором основаны все выводы работы - близость двух уровней в спектре системы, что позволяет говорить о более общем подходе к рассмотрению особенностей и свойств двухуровневых 
систем, нежели при использовании различных вариационных процедур, в частности метода ЛКАО [6-8].

Работа была поддержана грантами Международного соровского научного фонда (проект № 3F300) и Госкомитета Российской Федерации по высшему образованию (проект № 94-51-1080).

\section{ПРИЛОЖЕНИЕ}

Покажем, что значение параметра $q$, введенного в (13), не зависит от $\epsilon$. Для этого предположим наличие почти непроницаемого потенциального барьера, гранищы которого задаются уравнением

$$
V(x)=E .
$$

Два решения этого уравнения обозначим через $b_{1}$ и $b$ (считаем $b>b_{1}$ ) и будем предполагать, что точки $x_{0}, x_{1}$ лежат внутри этого интервала. Обозначим максимальное значение потенциальной энергии на этом участке как $V_{\max }$ и введем

$$
p=\sqrt{2 m\left(V_{\max }-E\right)} / \hbar .
$$

Используя новые функции

$$
S_{ \pm}(x)=\chi^{\prime}(x) \pm p \chi(x)
$$

представим уравнение Шредингера в форме

$$
S_{ \pm}^{\prime}(x) \mp p S_{ \pm}(x)=-2 m / \hbar^{2} L(x) \chi(x)
$$

где $L(x)=V_{\max }-V(x)$ всегда больше нуля в интервале $b_{1}<x<b$. Правую часть этого уравнения формально будем рассматривать как заданную функцию, что позволяет сразу же записать его решение для функций $S_{ \pm}(x)$. Выражая $\chi(x)$ через $S_{ \pm}(x)$ и используя приближенное равенство (т.к. справедливо условие (11))

$$
\chi_{0}(b) \approx \chi_{1}(b)
$$

(пусть $b-z>z-b_{1}$ ), получим для $q$ следующее выражение:

$$
q=p \frac{1-\frac{4 m}{\hbar^{2}} \exp [-p(b-z)] \int_{z}^{b} d x L(x) \operatorname{sh}[p(b-x)] f(x-z) / p}{1-\frac{4 m}{\hbar^{2}} \exp [-p(b-z)] \int_{z}^{b} d x L(x) \operatorname{sh}[p(b-x)] g(x-z)}
$$

где использовано условие $p(b-z) \gg 1$.

Если считать $L(x)$ малой добавкой, функции $f(x-z)$ и $g(x-z)$ достаточно взять в нулевом приближении:

$$
f(x-z)=\operatorname{ch}[p(x-z)], \quad g(x-z)=\operatorname{sh}[p(x-z)] / p .
$$


Подстановка их в выражение для $q$ дает оценку $q \leq p$, поскольку всегда $L(x) \geq 0$. В силу произвольности функции $L(x)$ отсюда следует, что максимальное значение параметра $q$, равное $p$, достигается в случае постоянного потенциала $V(x)=V_{\max }$.

Аналогично можно установить нижнюю границу возможных значений параметра $q$. Определим две точки на оси $x: x=a_{1}$ и $x=a\left(a_{1}>b_{1} ; a<b\right)$, такие, что $V\left(a_{1}\right)=V(a)$, $V(x)>V(a)$, и потребуем, чтобы произведение $k \cdot\left(a-a_{1}\right)$ было максимальным. Величину $k$ определим как $\sqrt{2 m(V(a)-E)} / \hbar$ и будем считать, что $k(a-z) \gg 1$ (условие непроницаемости барьера). Снова получим неявное решение уравнения Шредингера и приравняем волновые функции в точке $x=a$. Считая, что отклонение потенциальной энергии от значения $V(a)$ мало:

$$
\Lambda(x)=V(x)-V(a) \ll V(a)
$$

получим для параметра $q$

$$
q=k\left(1+\frac{4 m}{\hbar^{2} k} \exp [-k(a-z)] \int_{z}^{a} d x \Lambda(x) \operatorname{sh}[k(a-x)] \exp [-k(x-z)]\right)
$$

Поскольку $\Lambda(x)$ - неотрицательная функция произвольного вида, очевидно, что $q$ всегда больше $k$, а значение $q=k$ при $\Lambda=0$ является минимально возможным.

Таким образом, окончательно находим, что область изменения величины $q$ задается следуюшими неравенствами:

$$
k \leq q \leq p
$$

\section{Список литературы}

[1] В.В. Капаев, Ю.В. Копаев, Н.В. Корняков // Письма в ЖЭТФ. 1993. Т. 58. С. 901.

[2] В.Е. Житомирский // Письма в ЖЭТФ. 1992. Т. 55. С. 657.

[3] F. Stern, S. das Sarma // Phys. Rev. 1984. V. B30. P. 840.

[4] М.Г. Веселов. Элементарная квантовая теория атомов и молекул. М.: Физматгиз, 1962.

[5] Ч. Коулсон. Валентность. М.: Мир, 1965.

[6] J.W. Corbett // Am. J. Phys. 1963. V. 31. P. 521.

[7] W.A. Phillips // Proc. Roy. Soc. 1970. V. A319. P. 565.

[8] Л.Д. Ландау, Е.М. Лившиц. Квантовая механика. М.: Наука, 1989.

Нижегородский государственный университет

Поступила в редакцию 11.VIII.1995 г.

\section{V.A. Burdov \\ SOME PROPERTIES OF STATIONARY STATES OF TWO-LEVEL SYSTEMS}

It is shown that some function $J(x)$ is a constant at any point on $x$-axis, when a change of the potential energy of two-level system is arbitrary. The estimations of the LCAO method's accuracy are presented for the wave functions of a one-dimensional two-level system. 